\title{
Integration and innovation: first issue of cardiovascular intervention and therapeutics
}

\author{
Tetsuya Sumiyoshi
}

Published online: 8 December 2009

(C) Japanese Association of Cardiovascular Intervention and Therapeutics 2009

It is our great pleasure to announce the inaugural issue of cardiovascular intervention and therapeutics (CVIT), the official journal of the Japanese Association of Cardiovascular Intervention and Therapeutics (JACVIT). In 2009, JACVIT was established by integrating two major existing medical societies: the Japanese Society of Interventional Cardiology (JSIC) and the Japanese Association of Cardiovascular Catheterization and Therapeutics (JACCT). Concurrently with this organizational change, the two Japanese journals published by JSIC and JACCT were also merged to create a new English-language journal, CVIT, together with a Japanese-language version of the journal. CVIT starts with Vol. 25 No. 1, as it follows in sequence from the former journal.

Cardiovascular interventions in Japan have achieved a level of excellence rivaling that of other nations in the world, with many outstanding advanced techniques and results. In particular, Japanese investigators have led the world in the development of a number of techniques and technologies and have generated clinical trial results that have a major impact worldwide. These achievements will be most meaningful if they are disseminated globally to be evaluated by fellow researchers overseas engaged in the same field. The new English-language journal CVIT is indispensable to facilitate and promote this process. At the same time, we invite and encourage researchers from outside Japan to contribute to the journal, to exchange ideas, present their latest scientific and clinical findings, and compare their results with those of 6,000 JACVIT members who are receiving CVIT. Our mission is to make the journal a first-class international publication in this field.

CVIT publishes original articles and review articles and accepts case reports that advance our existing knowledge. The contents of the journal are not restricted to a narrow definition of cardiovascular interventions and therapeutics, but have a wide scope covering basic research on diagnostic methods or procedures that are clinically relevant and potentially beneficial to patients, as well as translational research that bridges the bench to bedside. Another innovation seen in the new journal is the introduction of Online First, which publishes accepted articles promptly in electronic form before they appear in printed form. This allows the latest information to be available to readers without the delay inherent in the production process of the printed issue. The new journal also features a completely novel cover design with original graphics.

With the help of the publisher, the editorial team is working toward applying for citation by Medline/PubMed Index and Science Citation Index (that is, to have an "impact factor"). With your valuable contributions and our reviewers' and editors' assistance, we will together make CVIT a top-ranked international journal.

Tetsuya Sumiyoshi, M.D., Ph.D. Editor-in-Chief

T. Sumiyoshi $(\square)$

Sakakibara Heart Institute, Tokyo, Japan

e-mail: tsumiyo@shi.heart.or.jp 